\title{
Cadmium-based Coordination Polymer Nanorods and Microblocks as Anode Materials for Lithium-Ion Batteries
}

\author{
Yanyong Sha ${ }^{1}$, Changdong Shi ${ }^{1}$, Yuanrui Gao ${ }^{2}$, Hao Wen ${ }^{1}$, Hongren Rong ${ }^{1}$, Hong-Jiang Liu ${ }^{2, *}$, \\ Qi Liu ${ }^{1,3, *}$ \\ ${ }^{1}$ School of Petrochemical Engineering and Jiangsu Key Laboratory of Fine Petro-chemical \\ Technology, Changzhou University, 1 Gehu Road, Changzhou, Jiangsu 213164, P. R. China \\ ${ }^{2}$ Department of Chemistry, College of Science, Shanghai University, No. 99 Shangda Road, Shanghai, \\ 200444, P. R. China. \\ ${ }^{3}$ State Key Laboratory of Coordination Chemistry, Nanjing University, Nanjing, Jiangsu 210093, \\ China \\ *E-mail: liuqi62@163.com, liuhj@ shu.edu.cn
}

doi: $10.20964 / 2019.02 .14$

Received: 21 July 2018 / Accepted: 12 September 2018 / Published: 5 January 2019

Layered cadmium-based coordination polymer $\left(\left[\mathrm{Cd}\left(4,4^{\prime}-\right.\right.\right.$-bpy $\left.\left.)(\mathrm{tfbdc})\left(\mathrm{H}_{2} \mathrm{O}\right)_{2}\right], \mathrm{Cd}-\mathrm{LCP}\right)$ nanorods and microblocks were synthesized by a facile hydrothermal method $\left(4,4^{\prime}\right.$-bpy $=4,4^{\prime}$-bipyridine, $\mathrm{H}_{2} \mathrm{tfbdc}=$ tetrafluoroterephthalic acid). The Cd-LCP were characterized by IR spectrum, elemental analysis, powder X-ray diffraction, thermogravimetric analysis, scanning electron microscope (SEM), transmission electron microscope (TEM), and the Brunauer-Emmett-Teller (BET) surface. As an anode material of lithium-ion batteries, the Cd-LCP nanorods and microblocks showed a higher reversible capacity and an excellent cyclic stability, maintaining $231 \mathrm{~mA} \mathrm{~h} \mathrm{~g}^{-1}$ after 50 cycles at $50 \mathrm{~mA} \mathrm{~g}^{-1}$. Our results further confirm that searching for anode materials of lithium-ion batteries from $2 \mathrm{D}$ coordination polymer is an effective route.

Keywords: cadmium compound; coordination polymer; lithium-ion batteries; electrode material

\section{FULL TEXT}

(C) 2019 The Authors. Published by ESG (www.electrochemsci.org). This article is an open access article distributed under the terms and conditions of the Creative Commons Attribution license (http://creativecommons.org/licenses/by/4.0/). 